\title{
Unterstützte Testausführung für automatisierte Fertigungsanlagen durch Verknüpfung von Testmanagement und E-CAD sowie Einbindung von Augmented Reality
}

\author{
S. Ziegltrum, Prof. Dr.-Ing. B. Vogel-Heuser, \\ Technische Universität München; \\ J. Frank, Zuken E3 GmbH; \\ F. Hasselfeld, CIM-BASE GmbH Consulting und Engineering; \\ H. Gröbl, DORST Technologies GmbH \& Co. KG; \\ C. Botzenhardt, MULTIVAC Sepp Haggenmüller GmbH \& Co. KG; \\ B. Werner, CODESYS GmbH
}

\section{Kurzfassung}

Im Bereich der variantenreichen Maschinen und im Sondermaschinenbau stellen Arbeiten an den häufig heterogenen und stark unterschiedlichen Anlagen besonders hohe Ansprüche an die Fachkompetenz des eingesetzten Inbetriebnahme- und Wartungspersonals. Zudem erfordern gesetzliche Änderungen die jährliche Überprüfung z.B. der Anlagensicherheit beim Kunden. Um diese gestiegenen Belastungen an das Personal zu bewältigen, sowie die Durchführung der Tests auch für weniger spezialisiertes Personal zu ermöglichen, bedarf es innovativer technischer Methoden zur Unterstützung des Anwenders über den gesamten Testerstellungssowie Testdurchführungsworkflow hinweg. Grundlage für die Erstellung und Ausführung von Sicherheitstests sind Engineering-Dokumente wie Stromlaufpläne, Unterlagen zum Sicherheitsnachweis sowie Sicherheitsverschaltungen.

In diesem Beitrag stellen wir Methoden vor, die ein breites Spektrum an Personal vor Ort dazu befähigen, periodisch wiederkehrende Tests der Sicherheit von automatisierten Fertigungsanlagen selbstständig sowie intuitiv und interaktiv angeleitet auszuführen. Zur Durchführung der Prüfungen ist es notwendig, Testpersonal z.B. mittels Augmented Reality bei der Orientierung an der Anlage zu unterstützen. Hilfestellung für das Personal besteht dabei beispielsweise in einer vereinfachten Navigation in den Anlagenplänen sowie der Anlage selbst, z.B. mit Hilfe des Betriebsmittellageplans, durch Anbindung des Testmanagers an das E-CAD-System. Wichtiger Baustein der Benutzerführung ist dabei eine effiziente und eindeutige Identifikation und Auffindung von für die Teststimulation bzw. Testobservation benötigten Anlagenkomponenten. 


\section{Einführung und Stand der Technik}

Die anhaltend fortschreitende Spezialisierung im Maschinen- und Anlagenbau - getrieben durch den Kundenwunsch nach immer stärker personifizierten Produkten und somit immer flexibleren Produktionsanlagen - stellt die Hersteller von Sondermaschinen vor große neue Herausforderungen. Die steigende Anzahl an Anlagenvarianten bedingt die drastische Reduzierung der Losgrößen einiger Sondermaschinenbauer bis zu einer Regellosgröße von kaum mehr als 1. Gleichzeitig ordnen sich über $60 \%$ der deutschen Maschinen- und Anlagenbauer als Hersteller des Premiumpreissegments ein. [1] Um diesen Ruf und die damit verbundenen hohen Produktkosten zu rechtfertigen, stehen die Hersteller unter Druck, ebenso überdurchschnittliche Qualität und Zuverlässigkeit ihrer Anlagen zu erreichen.

Nach Befragung mehrerer Weltmarkführer im Bereich des Sondermaschinenbaus konnten unter anderem folgende Herausforderungen identifiziert werden: Der hohe Anteil an unterschiedlich aufgebauten Anlagenvarianten, insbesondere innerhalb der Schaltschränke, macht die Rationalisierung der Fertigung und den Einsatz günstigerer und weniger qualifizierter Mitarbeiter in der Qualitätssicherung schwierig [2]. In der Praxis häufig anzutreffende 200\%-Checklisten für Anlagentests sind anfällig z.B. für Fehler bei der Selektion zutreffender Testfälle. Die Erstellung von variantenspezifischen Anleitungen für jede einzelne Anlage sowie die Schulung von Wartungsmitarbeitern der Endkunden stellen wiederum einen hohen Aufwand für die Anlagenhersteller dar. Viele Arbeiten von der Montage über die Inbetriebnahme, der Fehlersuche sowie regelmäßige Wartungen und Tests können derzeit häufig nur durch teure, hochspezialisierte Techniker vorgenommen werden. [3]

Eine mögliche Lösung dieser Probleme ist die Anleitung des Personals mittels intuitiver Softwaretools auf Grundlage von Dokumenten der Elektrokonstruktion. Da formelle Stromlaufpläne für jede Sondermaschine durch die Hersteller bereits variantenspezifisch erstellt werden müssen, stellen diese die ideale Datenbasis für intelligente, softwaregestützte Assistenzsysteme dar, um Techniker u.a. beim Test der Sicherheitseinrichtungen zu unterstützen. Auf Grund ihrer durch Normen fest vorgegebenen Struktur, sowie der umfangreichen API moderner E-CAD-Lösungen können diese Daten zuverlässig maschinell interpretiert, aufbereitet und somit für Benutzer unterschiedlicher Qualifikation sowie Aufgaben bereitgestellt werden. Die Selektion variantenspezifischer Tests mittels Schaltplänen und die Anleitung von Inbetriebnehmern durch Verknüpfung von Schaltschrankaufbau- und Betriebsmittellageplänen mit Augmented Reality (AR) stellen dabei einen vielversprechenden Lösungsansatz dar. 
Die Eignung von Augmented Reality zur Bereitstellung von kontextsensitivem Wissen wurde bereits in einer Vielzahl von Szenarien erprobt. Sowohl die Bereitstellung von prozeduralem Wissen sowie Sachwissen kann mittels Augmented Reality realisiert werden. Die unmittelbare Verknüpfung des Wissens mit dem Kontext auf Grund der unmittelbaren physischen Nähe erleichtert auch weniger qualifizierten Nutzern das Verständnis der gewünschten Informationen. [4] Auch wenn Augmented Reality im Bereich der mechanischen Montage und Wartung eingehend erforscht und umgesetzt wurde, mangelt es bisher an deren Übertragung auf das Testen elektrischen Sicherheitstechnik. Besonders die Gewinnung der für die Verknüpfung zwischen AR und Realität notwendigen geometrischen Informationen aus dem E-CAD stellen hier eine Herausforderung dar. Ebenso müssen die spezifischen Umweltbedingungen des Maschinen- und Anlagenbaus für den Einsatz von Augmented Reality berücksichtigt werden. Selbst etablierte Softwarehersteller wie EPLAN oder AUCOTEC bieten keine entsprechende Unterstützung des Testpersonals im Bereich der variantenspezifischen Inbetriebnahme oder geführten Fehlersuche.

Der nachfolgende Beitrag präsentiert eine Möglichkeit, mittels innovativer Kombination von klassischen Testanleitungen, elektronischen Dokumenten des E-CAD sowie modernen Konzepten der Augmented Reality Benutzer unterschiedlicher Qualifikation unter anderem zur Durchführung von Sicherheitstests an variantenreichen automatisierten Produktionsanlagen zu befähigen. Es wird ein Konzept erarbeitet, wie Großteils bereits vorhandene Informationen des E-CAD mittels Augmented Reality für unterschiedliche Einsatzzwecke flexibel verfügbar gemacht werden können. Zudem wird vorgestellt, wie noch fehlende Voraussetzungen für die beabsichtigte Anwendung von AR effizient und einfach geschaffen werden können.

\section{Industrielle Rahmenbedingungen zur Anwendung von Augmented Reality}

In diesem Kapitel sollen besondere Rahmenbedingungen und Anforderungen für den Einsatz von Augmented Reality zur Testanleitung variantenreicher automatisierter Produktionsanlagen identifiziert werden. Es wird ein typisches Format von Sicherheitstests vorgestellt. Anschließend werden besondere Voraussetzungen erläutert, die für den zuverlässigen Einsatz von Augmented Reality im industriellen Umfeld notwendig sind.

\subsection{Anwendungsfall}

Wie bereits beschrieben, liegen Testanleitungen zum Test der Sicherheitseinrichtungen in der Regel als sogenannte 200\%-Pläne vor, welche Tests für alle möglichen Ausprägungen von 
Varianten beinhalten. Es ist bisher Aufgabe des Sicherheits- oder Testingenieurs, diejenigen Testfälle auszuwählen, die für eine spezifische Anlagenvariante zutreffend sind.

Die Pläne haben meistens die Form einer Checkliste oder einer Matrix, in der alle Permutationen der ausgelösten Sicherheitssensoren den abgesicherten Aktoren sowie erwarteten Reaktionen gegenübergestellt sind. Nach Auswertung von Dokumenten zum Sicherheitstest einiger führender Sondermaschinenhersteller lassen sich die einzelnen Testfälle in vier Phasen gliedern, welche derzeit meist textuell in den Testanweisungen hinterlegt sind:

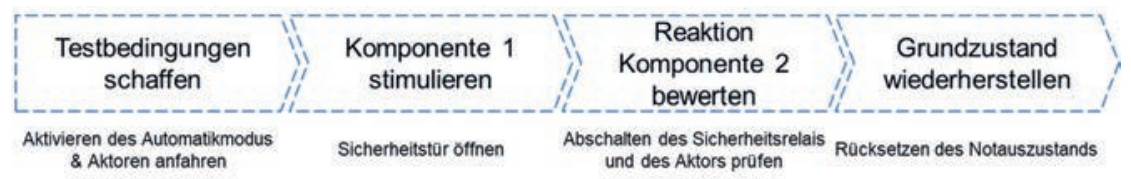

Bild 1: Phasenmodell eines Testfalls einschließlich beispielhafter Testschritte

Die Identifikation der Testkomponenten basiert dabei meist auf Betriebsmittelkennzeichen nach EN 81346, die entsprechend firmeninterner Konvention vergeben werden und über verschiedene Anlagenvarianten hinweg konsistent sind. Auch wenn die Bezeichnungen der Komponenten eindeutig sind, erschwert diese abstrakte Lesart laut Befragungen industrieller Experten besonders für Anlagenbediener ohne elektrotechnisches Fachwissen das einfache Verständnis der durchzuführenden Testanweisungen. Andererseits können diese Bezeichnungen auch die Ausgangsbasis für die automatische Verknüpfung von Tests zu den Dokumenten des E-CAD bilden.

\subsection{Industrielle Rahmenbedingungen}

Zur zielgerichteten Entwicklung praktisch anwendbarer und nutzbringender Lösungen ist eine frühzeitige Betrachtung der Rahmenbedingungen des beabsichtigten Nutzungsumfelds von großer Bedeutung. Dabei müssen alle Einflüsse und Nutzer des Systems berücksichtigt werden. Dies beinhaltet erstens Anforderungen durch Mitarbeiter zur Formulierung der Testanweisungen, durch Mitarbeiter der Elektrokonstruktion als auch durch späteres Testpersonal. Zweitens müssen organisatorische Rahmenbedingungen wie beispielsweise rechtliche Einschränkungen und Forderungen betrachtet werden. Drittens existieren technische Rahmenbedingungen, z.B. der AR-Geräte, der Automatisierungsanlage selbst und des Anlagenstandorts. Um die Anwendbarkeit der erarbeiteten Lösungen im industriellen Umfeld anwenden zu 
können, ist eine sorgfältige Analyse des späteren Einsatzumfeldes unabdingbar. Die folgenden Abschnitte beschreiben deshalb relevante Restriktionen des industriellen Anwendungsgebiets in dem das entwickelte System eingesetzt werden soll.

\section{Rahmenbedingungen der Anwender}

Die Durchführung der periodischen Wartung von Produktionsanlagen soll für eine möglichst große Anwendergruppe ermöglicht werden. Dies bedeutet eine Nutzung der Augmented Reality Anwendung sowohl durch elektrotechnisch ausgebildetes Wartungspersonal als auch durch Anlagenbediener ohne technischen Hintergrund.

\section{Organisatorische Rahmenbedingungen}

Als Konsequenz der unterschiedlichen Ausbildungen folgen rechtliche Restriktionen, welche Eingriffe in die Anlage das Testpersonal ausführen darf. Die Sicherheitstechnik der Automatisierungsanlagen muss für die Erweiterung des Anwendergebiets also dahingehend ausgelegt sein, dass die Durchführung der Tests auch für ungeschultes Personal rechtlich zulässig ist. Weiterhin unterliegt der Test von Sicherheitseinrichtungen strengen rechtlichen Auflagen. Tests müssen vollständig ausgeführt, regelmäßig wiederholt und vollständig dokumentiert werden. Die Konsistenz der 200\%-Checklisten mit den variantenspezifischen Konstruktionsdokumenten muss deshalb entwicklerseitig sichergestellt sein.

\section{Technische Rahmenbedingungen}

Während der Einführung von neuen Technologien ist mit initialem Zusatzaufwand zu rechnen, z.B. durch Anpassung von Dokumenten oder eventuell notwendigen Änderungen der Hardware. Diese sollen aber geringgehalten und idealerweise nur einmalig notwendig sein. Je nach Standort der Anlage ist auch im späteren Betrieb mit unterschiedlichen technischen Herausforderungen für die Funktionalität der AR zu rechnen. Optische Trackingsysteme können durch Verschmutzung der Anlage oder mangelhafte Beleuchtung der Werkshalle, GPS- und kompassbasierte Trackingsysteme durch Metall in der näheren Umgebung unbrauchbar gemacht werden. Die eingesetzte AR-Plattform muss zudem physisch für die Einsatzbedingungen geeignet sein. Dies beinhaltet Widerstandsfähigkeit gegenüber Umwelteinflüssen als auch eine ergonomische Benutzbarkeit durch den Anwender. 


\subsection{Anforderungen zur Testanleitung mittels Augmented Reality}

Auf Grundlage der beschriebenen Rahmenbedingungen wurden vier Anforderungen abgeleitet, die von der Applikation erfüllt werden sollen. Die Anforderungen werden wie folgt beschrieben:

1. Vollständigkeit und Validität der Sicherheitstests. Zentrale Voraussetzung für die Funktionalität ist die korrekte Durchführung periodisch wiederkehrender Sicherheitstests von variantenreichen Automatisierungsanlagen. So muss sichergestellt sein, dass alle für eine Anlagenvariante relevanten Tests vom Testpersonal valide durchgeführt werden können.

2. Ergonomische Anleitung der Tests. Bei der Testanleitung sollen auch Benutzern ohne tiefgreifendes Fachwissen alle für den Test notwendigen Informationen automatisiert aufbereitet und zur Verfügung gestellt werden. Die Hardwareplattform der AR muss dem Benutzer zudem die notwendige physische Flexibilität während der Durchführung gewähren. Dies beinhaltet u.a. die lokale Verarbeitung aller Daten ohne Notwendigkeit einer Netzwerkverbindung oder eines Stromanschlusses.

3. Zuverlässige Funktionalität der Augmented Reality im industriellen Umfeld. Zur Durchführung valider Tests durch Personal mit geringem technischen Fachwissen ist die eindeutige und zuverlässige Identifikation aller am Test beteiligter Komponenten zwingend notwendig, auch unter typischerweise fordernden Umwelteinflüssen eines industriellen Umfelds.

4. Minimaler Aufwand bei der Einführung des Systems. Um AR nutzbringend einsetzen zu können, muss der initiale Aufwand für die Einrichtung sowie die Kosten für die Vorbereitung jeder realen Anlage so gering wie möglich gehalten werden.

\section{Architekturentwurf eines interaktiven AR-Frameworks}

Für die Bereitstellung der Kernaufgabe der Augmented Reality, der eindeutigen Identifikation von für den aktuellen Testschritt relevanter Anlagenbauteile innerhalb und außerhalb des Schaltschranks durch das Testpersonal, sind mehrere zusammenwirkende Module notwendig. Die Komponenten der AR-Applikation werden im Folgenden näher beschrieben.

- Mittels Tracking wird AR dazu befähigt, Objekte im Umfeld des AR-Geräts zu identifizieren, deren relative Position zu bestimmen und dynamisch zu aktualisieren. Die Präzision des Tracking-Moduls muss mindestens der kleinsten Strukturgröße entsprechen, deren Position bestimmt werden soll. Auch muss die Qualität des Trackings kon- 
tinuierlich überwacht und dem Nutzer mitgeteilt werden, sobald die Präzision des Trackings nicht mehr für die Identifikation von Anlagenkomponenten ausreichend sichergestellt werden kann. [5]

- Zur Kommunikation mit dem Testpersonal dient ein HMI. Dies ermöglicht es dem ARSystem den Benutzer über notwendige Testschritte zu instruieren, den Zustand des Trackings anzuzeigen und die zusätzlichen Informationen der Augmented Reality in die Umwelt zu projizieren. In die Gegenrichtung erhält aber auch der Nutzer die Möglichkeit, z.B. Testergebnisse an das Testmanagement zu melden.

- Über eine weitere Schnittstelle wird die Kommunikation zwischen AR und Testmanagement etabliert. Dieses teilt der AR mit, welche Testschritte als nächstes durchzuführen sind und über welches Betriebsmittelkennzeichen das Bauteil im Kontext des aktuellen Testschritts verfügt. Andererseits können über das Interface z.B. Testresultate an das Testmanagement zurückgegeben und neue Anweisungen angefordert werden.

- Die zur Benutzerführung notwendigen Informationen über Schaltschrankidentifikation und -aufbau sowie weitere Daten über den Aufbau der Anlage erhält das AR-System über eine E-CAD-Schnittstelle. Sind Komponenten des Tests nicht im Schaltschrank, sondern außerhalb an der Anlage verbaut, kann dem Benutzer die Lage des Bauteils alternativ schematisch im Betriebsmittellageplan angezeigt werden.

\section{Technische Umsetzung und verwendete Hardware}

Die beschriebenen Module wurden im Hinblick auf verfügbare industrierealistische Evaluationsdaten prototypisch umgesetzt und eingehend erprobt. Im Folgenden werden die jeweiligen Möglichkeiten zur Umsetzung der Module beschrieben und gegeneinander abgewogen.

\subsection{Implementierung der E-CAD-Schnittstelle}

Zwei in Deutschland weit verbreitete Entwicklungsumgebungen für die Elektroprojektierung sind $\mathrm{E}^{3}$.series der Firma Zuken sowie EPLAN des gleichnamigen Herstellers. Beide bieten umfassende proprietäre APIs zur digitalen Kommunikation mit den jeweiligen Softwareumgebungen sowie dazugehörige Datenmodelle. Auf Grund der Verfügbarkeit industrierealistischer Evaluationsprojekte und der umfangreichen Schnittstellendokumentation fiel die Wahl auf die Entwicklung mit E3.series. Die Kommunikation erfolgt mit Hilfe eines REST-Deamons als Middleware zur COM-Schnittstelle von $\mathrm{E}^{3}$.series. $\mathrm{E}^{3}$.series ist ebenfalls dazu in der Lage, detaillierte Geometrien sowie Drahtgittermodelle der anzuzeigenden Komponenten für die AR zur Verfügung zu stellen. 


\subsection{Schnittstelle zum Testmanagement}

Das Testmanagement sowie die Selektion variantenspezifischer Testfälle mittels BMK wurde prototypisch mit MySQL und C\# umgesetzt. Auch hier sichern ein modularer Aufbau sowie eine standardisierte REST-Schnittstelle die Kompatibilität zu anderen kommerziellen Systemen und einer Vielzahl an weiteren Einsatzszenarien der AR.

\subsection{Umsetzung des HMI}

Für die Realisierung des HMI können drei Gruppen von Implementierungen unterschieden werden: Entwicklung einer neuen Anwendung mittels vorgefertigter Funktionen und SDKs von Grund auf, dem Einsatz spezialisierter, teils kommerzieller Tools sowie der Anpassung diverser kostenpflichtiger und kostenfreier 3D-Engines. [4] Um die spätere Verwendung des Systems im industriellen Umfeld zu erleichtern, sollten weitestgehend freie Softwarekomponenten eingesetzt werden. Eine weitere Einschränkung des Lösungsraums ist durch die durch das ECAD-System erzwungene Kompatibilität mit Microsoft Windows als Betriebssystem erfolgt. Ebenso muss das HMI einfach mit den anderen Funktionsmodulen verbunden werden können, weshalb die Wahl auf Grund der umfangreichen Bibliothek an Plugins und SDKs auf die UnityEngine gefallen ist. Die Implementierung der Anwendung ist für die Unified Windows Platform (UWP) erfolgt. Dies ermöglicht zum einen den Betrieb gemeinsam mit Testmanagement und E-CAD beispielsweise auf einem Laptop. Darüber hinaus kann die AR-App durch Nutzung der REST-Schnittstellen aber auch einfach auf anderen UWP-Geräten wie Windows Phones oder einer Microsoft HoloLens betrieben werden. Für den Test des Demonstrators ist die Wahl auf Grund des Touchbildschirms, der rückseitigen Kamera sowie der guten Portabilität auf ein Surface Book von Microsoft gefallen.

Die Interaktion mit dem Benutzer erfolgt in zwei Richtungen. Durch Überlagerung des Livebildes z.B. eines Schaltschranks mit geometrischen Markierungen können bestimmte Komponenten für den Benutzer hervorgehoben werden. Zusätzlich wurde ein Head-up-Display realisiert, das dem Benutzer textuell sukzessive Anweisungen zum weiteren Testvorgehen einblenden kann. Über den Touch-Bildschirm kann der Benutzer aber auch mit der AR interagieren und so z.B. bestimmte Komponenten an der Anlage durch simple Berührung auswählen und weiterführende Informationen zu erhalten. Auch Statusabfragen oder Dialoge wären so realisierbar. Bei Einsatz einer AR-Brille könnte z.B. Interaktion mittels Sprache oder Gesten eingesetzt werden. 


\subsection{Tracking}

Zur Erfüllung der beabsichtigten Aufgaben des Trackings (Abschnitt 3) ist die Erfüllung vieler resultierender Anforderungen notwendig. Für das Tracking kann zwischen aktiven und passiven Methoden unterschieden werden. Eine Kombination mehrerer Ansätze ist je nach Komplexität der eingesetzten Software und verfügbarer Rechenleistung ebenfalls möglich.

Aktives Tracking erfordert jeweils die Anbringung von aktiven Komponenten wie z.B. Infrarotoder Magnetfeldemittern. Die Notwendigkeit von Sichtkontakt zu den Trackern würde für eine große Automatisierungsanlage oder Anlagen mit mehreren Schaltschränken auch den Einsatz von mehreren Sendern an jeder Anlage voraussetzen, was aus Kostengründen verworfen wurde. Tracking-Ansätze wie GPS und die Nutzung von Magnetfeldsensoren ist in geschlossenen Fertigungshallen und in der Nähe von Stromleitungen großer Stromstärke sehr fehleranfällig, weshalb auch diese Methoden zur Erstellung einer zuverlässigen AR-Applikation ausgeschlossen wurden. Nach Ausschluss ungeeigneter Lösungen fiel die Wahl auf einen hybriden Ansatz von passiven optischen Markern und der Bewegungsverfolgung mittels Inertialsensorik. [5]

Zur Implementierung der Funktionalität passiver optischer Marken stehen mehrere populäre freie Bibliotheken zum Einsatz in Kombination mit der Unity-Engine zur Verfügung. Neben dem AR Toolkit und Wikitude SDK fiel die Wahl nach prototypischer Erprobung auf die Bibliothek VuForia. VuForia nutzt 2D-Codemuster, so genannte VuMarks, welche als Orientierungspunkt der AR im Schaltschrank angebracht werden. Abhängig vom größten Abstand zwischen getracktem Betriebsmittel und VuMark muss die Größe des Markers entsprechend gewählt werden. Die Platzierung des Markers kann wahlweise bereits im E-CAD (Bild 2) oder bei Bedarf erst auf der Baustelle erfolgen. Zu beachten ist, dass auch beim Einsatz von passiven optischen Trackern der Sichtkontakt zwischen Marker und AR-Gerät zwingend notwendig ist. Darüber hinaus können Probleme durch unzureichende Beleuchtung oder Kontrast sowie bei Verschmutzung des Markers auftreten. 


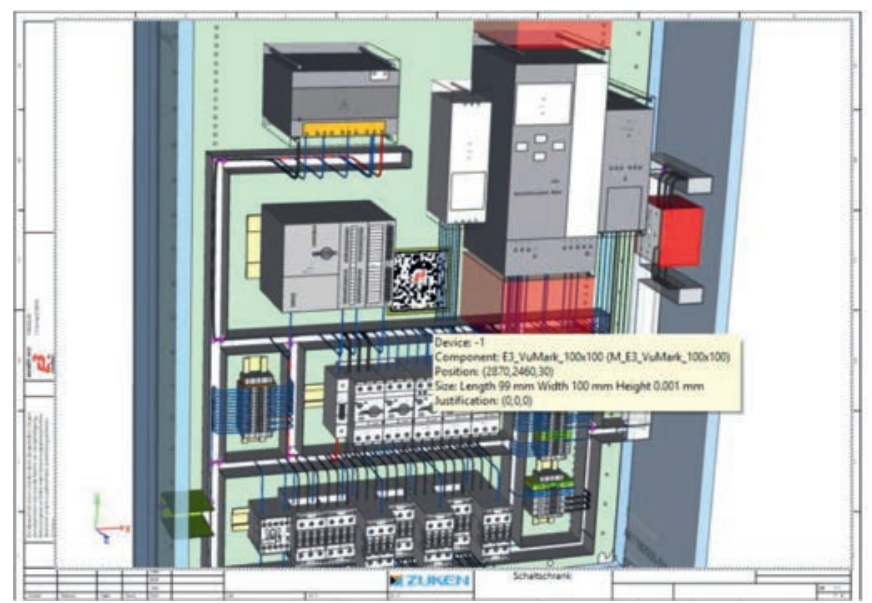

Bild 2: Platzierung des VuMarks mittels E-CAD

\section{Evaluation}

Die entwickelte AR-Anwendung wurde unter unterschiedlichen Umweltbedingungen wie Lichtverhältnissen und Nutzergruppen eingehend getestet. Bei einer Montageplatte von $1 \mathrm{~m} \times 2 \mathrm{~m}$ Größe und mittiger Platzierung eines 0,1m x 0,1m VuMarks (Bild 3) konnten selbst Reihenklemmen am Rand der Montageplatte noch zuverlässig markiert werden. Die Generierung und Anbringung der VuMarks erfordert lediglich einen Drucker, weshalb die Marker z.B. bei Verschmutzung leicht ausgetauscht werden können. Die initialen Kosten pro Anlage sind dadurch minimal. Als Schwachpunkt des aktuellen Vorgehens wurde die Summierung von Positionierungsfehlern der VuMarks identifiziert. Da der Trackingalgorithmus den Koordinatenursprung einzig anhand des VuMarks bestimmt und die Bauteilpositionen anhand der Daten des E-CAD berechnet, pflanzen sich diese Fehler an alle montierten Bauteile derselben Montageplatte fort. Bei der Platzierung des VuMarks muss deshalb besonders sorgfältig vorgegangen werden. Eine Verbesserung wäre beispielsweise der Aufdruck des VuMarks auf eine Komponente zur Hutschienenmontage, welche sehr viel leichter exakt platziert werden könnte.

Mittels konsequentem Einsatz von grafischen Markierungen in AR und Betriebsmittellageplan benötigt der Nutzer keinerlei Wissen im Umgang mit BMKs. Für die Unterscheidung mehrerer Schaltschränke an einer Anlage wurde der Name des E-CAD-Projekts sowie die BMK der Montageplatte mit in den VuMarks codiert. Auf diese Weise erhält der Benutzer Feedback, ob 
er sich am richtigen Schaltschrank der richtigen Anlage befindet. Liegt das anzuzeigende Bauteil außerhalb der aktuellen Kameraperspektive wird der Nutzer durch ein Pfeilsymbol am Bildschirmrand in die entsprechende Richtung verwiesen (Bild 4). Eine kontinuierliche Überwachung der Präzision des Trackings einschließlich einer visuellen Warnung an den Benutzer sichert die Fehlerfreiheit der Bauteilmarkierungen ab. Durch den Einsatz der AR-Anwendung auf einem Tablet war die Abarbeitung der Anlagentests zu jeder Zeit ergonomisch möglich.

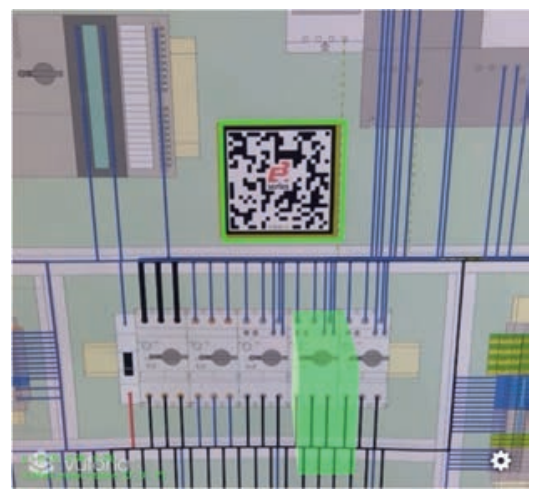

Bild 3: Tracking eines VuMarks und Markierung einer Komponente

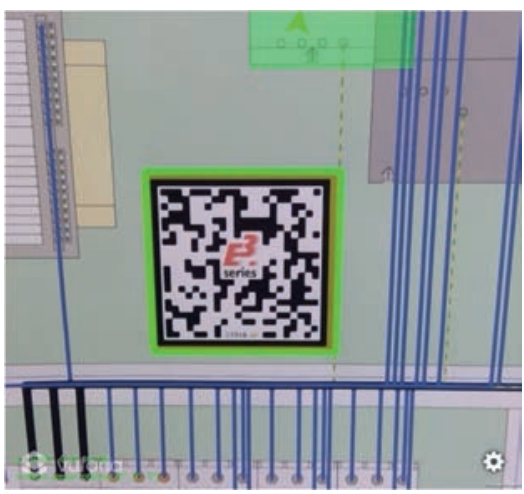

Bild 4: Markierung eines Bauteils außerhalb der Kameraperspektive

\section{Zusammenfassung und Ausblick}

Im vorliegenden Beitrag wurde ein Framework entwickelt, das die effiziente und ökonomische Durchführung von wiederkehrenden Sicherheitstests an variantenreichen Automatisierungsanlagen durch Testpersonal mit minimalem elektrotechnischem Hintergrund mit Hilfe von Augmented Reality ermöglicht. Ausgehend von einer Beschreibung derzeit typischer Testvorgänge wurde der Bedarf nach Softwareunterstützung für Testpersonal unterschiedlicher Qualifizierung aufgezeigt, analysiert und unter Berücksichtigung der Rahmenbedingungen Anforderungen an ein entsprechendes AR-System abgeleitet. Die Anforderungen wurden anschließend notwendigen Teilkonzepten einer möglichen Lösungsarchitektur gegenübergestellt. Nach Auswahl geeigneter Konzepte für die Anbindung von Testmanagement und E-CAD, zur Umsetzung des HMI sowie von Trackingmechanismen wurde ein Prototyp der Software an realistischen Prototypen evaluiert. Dabei wurde festgestellt, dass die so entstandene AR-Anwendung Abhilfe bei allen aufgezeigten Problemstellungen schaffen konnte. Für eine 
Schwachstelle des Prototypens wurden entsprechende Ansätze zur Behebung aufgezeigt. Mögliche künftige Weiterentwicklungen des Systems werden im Folgenden beschrieben.

Durch den Betrieb der AR-Anwendung auf einer Datenbrille könnte das Testpersonal physisch entlastet und die Ergonomie bei der Testdurchführung weiter verbessert werden. Durch Integration weiterer Bauteile wie beispielsweise Drähten, Kabeln oder der Pneumatik könnte die AR zudem zur Auffindung von Verdrahtungsfehlern erweitert werden. Des Weiteren könnte versucht werden, das Tracking im Schaltschrank z.B. durch den Einsatz mehrerer verteilter Marker auf einer Montageplatte zu verbessern. Durch Ausweitung der Konzepte auf Schaltpulte könnte die AR unerfahrene Benutzer dazu anleiten auch das HMI bei Tests der Anlage zu nutzen.

\section{Danksagung}

Die beschriebenen Konzepte und Ergebnisse wurden im Rahmen des Projekts EfiMA - „Effiziente Fehlersuche für sichere variantenreiche Maschinen- und Anlagenautomatisierung" - erarbeitet, welches durch die Bayerische Forschungsstiftung gefördert wird.

\section{Literaturangaben}

[1] J. Gernandt et al., "Zukunftsperspektive deutscher Maschinenbau," McKinsey Co. VDMA, p. 84, 2014.

[2] S. Ulewicz, B. Vogel-heuser, and I. S. Member, "Guided Semi-Automatic System Testing In Factory Automation," pp. 142-147, 2016.

[3] S. Rösch, S. Ulewicz, J. Provost, and B. Vogel-Heuser, "Review of Model-Based Testing Approaches in Production Automation and Adjacent Domains-Current Challenges and Research Gaps," J. Softw. Eng. Appl., vol. 08, no. 09, pp. 499-519, 2015.

[4] R. Palmarini, J. A. Erkoyuncu, R. Roy, and H. Torabmostaedi, "A systematic review of augmented reality applications in maintenance," Robot. Comput. Integr. Manuf., vol. 49, no. March 2017, pp. 215-228, 2018.

[5] E. Bostanci, N. Kanwal, S. Ehsan, and A. F. Clark, "User Tracking Methods for Augmented Reality," Int. J. Comput. Theory Eng., vol. 5, no. 1, pp. 93-98, 2013. 